\title{
COMPORTAMENTO VISUAL E DESENVOLVIMENTO MOTOR DE RECÉM-NASCIDOS PREMATUROS NO PRIMEIRO MÊS DE VIDA
}

\author{
VISUAL BEHAVIOR AND THE MOTOR DEVELOPMENT OF \\ PREMATURE NEWBORNS IN THE FIRST MONTH OF LIFE
}

\author{
Anna Paula Aca Ferreira ${ }^{1}$ \\ Raquel Costa Albuquerque ${ }^{2}$ \\ Aneide Rocha de Marcos Rabelo ${ }^{3}$ \\ Flavia Cabral de Farias ${ }^{4}$ \\ Rosana Cavalcanti de Barros Correia ${ }^{5}$ \\ Heloisa Gagheggi Ravanini Gardon Gagliardo ${ }^{6}$ \\ Ana Cláudia Vasconcelos Martins de Souza Lima ${ }^{7}$
}

Ferreira APA et al. Comportamento visual e desenvolvimento motor de recém-nascidos prematuros no primeiro mês de vida. Rev Bras Cresc e Desenv Hum 2011; 21(2): 335-343.

\section{RESUMO}

Objetivo: Caracterizar o comportamento visual e o desenvolvimento motor de RNPT com um mês de idade corrigida, nascidos na maternidade de um hospital escola. Método: Estudo descritivo, seccional no primeiro mês de idade corrigida em uma coorte de recém-nascidos prematuros, utilizando-se como instrumentos de avaliação o Método de Avaliação da Conduta Visual de Lactentes e o Alberta Infant Movement Scale (AIMS). Resultados: Observou-se que os recém-nascidos prematuros apresentaram respostas esperadas para as provas que avaliam o comportamento oculomotor e com frequências inferiores para a prova do sorriso, conforme o método de avaliação utilizado no estudo. Na avaliação motora, $25 \%$ apresentaram percentil abaixo de 5; 8,3\% com percentil 5; 8,3\% com percentil 10; $25 \%$ com percentil $25 ; 8,3 \%$ com percentil 50 e $25 \%$ com percentil 75 . Conclusões: Os resultados deste estudo demonstraram que mesmo em pequena amostra é possível observar atraso típico no desenvolvimento motor de lactentes prematuros quando se utiliza instrumento de avaliação adequado. Evidenciou-se que a idade gestacional pode ter influenciado o desempenho dos lactentes e que os recém-nascidos prematuros, mesmo com a idade corrigida, ainda estão aquém dos bebês a termo no que se refere às aquisições do desenvolvimento motor, indicando a necessidade de alerta ao desenvolvimento no percentil 10 ou abaixo, mesmo apresentando desempenho satisfatório no comportamento visual. A correção da idade foi um importante fator que norteou a compreensão das aquisições visuais e motoras de lactentes prematuros.

Palavras-chave: avaliação, desenvolvimento motor, função visual, recém-nascido prematuro.

1 Terapeuta Ocupacional do Instituto de Medicina Integral Prof. Fernando Figueira - IMIP, Pós-graduanda em Educação Especial.

2 Profa. Assistente do Departamento de Terapia Ocupacional / UFPE, Mestre em Saúde Materno Infantil pelo Instituto de Medicina Integral Professor Fernando Figueira/IMIP e Doutoranda em Saúde Materna Infantil pelo IMIP. E-mail: raquel.albuquerque@terra.com.br

3 Profa. Assistente do Departamento de Terapia Ocupacional / UFPE, Mestre em Saúde Materno Infantil pelo Instituto de Medicina Integral Professor Fernando Figueira /IMIP. E-mail: rabelo@nlink.com.br

4 Terapeuta Ocupacional da Associação de Assistência à Criança Deficiente - AACD, Mestranda do Programa de Pós Graduação em Saúde da Criança e do Adolescente / UFPE.E-mail:flaviafarias_flau@hotmail.com

5 Terapeuta Ocupacional, Docente do Departamento de Terapia Ocupacional/UNCISAL. Email: rosanarcbc@yahoo.com.br.

6 Terapeuta Ocupacional. Doutora e Docente da Pós Graduação da Faculdade de Ciências Médicas / UNICAMP. E-mail: heloisa@fcm.unicamp.br.

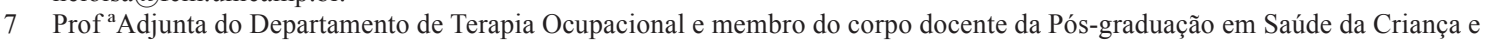
do adolescente/UFPE. Doutora em Nutrição/ UFPE. E-mail: anacvmsl@yahoo.com.br

Correspondência para: Anna Paula Aca Ferreira. Rua: Anísio Galvão-73, IPSEP - CEP: 51350-260 - Recife-PE. Fone celular: 081-92457333. Fone/Fax: 081-34713297. Email: annapaula_aca@hotmail.com

Trabalho realizado no Hospital das Clínicas da Universidade Federal de Pernambuco. Parte integrante do Projeto de Pesquisa. Fonte de financiamento: Conselho Nacional de Desenvolvimento Científico e Tecnológico (CNPQ). Edital Universal 2006/ 2008, $\mathrm{n}^{\circ}$ do processo: 483947/2006-7. 


\begin{abstract}
Objective: To characterize the visual behavior and the motor development of premature newborns (RNPT) with one month of age corrected, born in the maternity of a school hospital. Method: Descriptive study, sectional in the first month of age corrected in a cohort of a premature newborn, by using as instruments the Evaluation Method of the Visual behavior in newborns and the Alberta Infant Movement Scale (AIMS). Results: It was observed that the premature newborns presented expected answers for the tests that evaluate the oculomotor behavior and with lower frequencies for the test of smile, in agreement with the method of evaluation utilized in the study. In the motor evaluation (AIMS), 25\% presented below the percentage 5; $8.3 \%$ had percentage $5 ; 8.3 \%$ had percentage $10 ; 25 \%$ had percentage $25 ; 8.3 \%$ had percentage 50 and $25 \%$ had percentage 75 . Conclusion: The results obtained in this study showed that in despite of the small sample it was possible to observe a typical delay in the motor development in RNPT through an adequate instrument of evaluation. It was evident that the gestational age influenced the performance of the newborns and that the premature newborns even with the corrected age are still behind the full term babies regarding the acquisition of motor development, indicating the need of alert to the development at the percentile 10 or below, even presenting satisfactory performance in the visual behavior. Age correction was a significant factor facilitating in the understanding of the visual and motor acquisitions in RNPT.
\end{abstract}

Key words: assessment, motor development, premature newborns, visual function.

\section{INTRODUÇÃO}

A Organização Mundial da Saúde (OMS), em 1969 definiu que o recém-nascido com menos de 37 semanas de gestação é considerado como prematuro. ${ }^{1}$ Alves-Filho e Corrêa ${ }^{2}$ completam nos estudos que a classificação do recém-nascido prematuro, pode ser dividida em:pré-termo (menos de 37 semanas), pré-termo moderado (33 a 36 semanas), muito pré-termo ( 28 a 32 semanas) e pré-termo extremo (24 a 27 semanas).

No Brasil, a incidência de prematuridade entre os nascimentos tem variado de 5 a $15 \%$ e nos recém-nascidos pré-termo (RNTP) que sobrevivem, ${ }^{1,3}$ tem levado à maior incidência de distúrbios neurosensorimotores, quando comparados a bebês a termo, em decorrência da imaturidade funcional e estrutural nos siste$\operatorname{mas}^{4,5,6,7,8}$.

As crianças prematuras, no que concerne ao desenvolvimento visual, estão mais propensas a desenvolver alterações oftalmológicas como a retinopatia da prematuridade, o estrabismo e os erros de refração resultantes das lesões neurológicas, da toxidade pela luz e da privação sensorial no período de intenso desenvolvimento do sistema visual compreendi- do entre 28-40 semanas ${ }^{5,6,7}$ e com relação ao desenvolvimento motor, os prematuros, normalmente apresentam a alterações do tono muscular, reflexos primitivos ausentes ou diminuídos e movimentos espontâneos reduzidos, podendo apresentar atrasos na aquisição de controle da motricidade axial, apendicular e visuomotora ${ }^{3,9}$.

Desse modo, as aquisições visuais e motoras no primeiro semestre de vida de lactentes prematuros são fatores que interferem no aprendizado e desenvolvimento infantil e estudos relacionados a estas habilidades são pertinentes, já que a correlação das funções oculomotoras facilitam no ajuste e modificação da postura e nos movimentos dos membros superiores e inferiores ${ }^{7,10,11,12,13}$.

Além disso, fatores ambientais (ambiente físico, escolaridade dos pais, dinâmica familiar e situação econômica), exercem influência no desenvolvimento motor e cognitivo de crianças ${ }^{14}$, com conseqüências positivas e/ou negativas para o desenvolvimento infantil. ${ }^{15,16}$

No campo da terapia ocupacional, cada vez mais se evidencia a preocupação desses profissionais com a promoção do desenvolvimento infantil e com a prevenção de alterações no desenvolvimento ao longo da infância. Des- 
sa maneira, esses profissionais, especialmente treinados, têm utilizado diferentes instrumentos de avaliação para detectar precocemente alterações que possam interferir no repertório de desempenho funcional dessas crianças.

Assim, tanto na prática clínica como em pesquisa, com relação ao comportamento visual tem-se utilizado o Método de Avaliação da Conduta Visual de Lactentes, que foi estruturado com a finalidade de identificar possíveis sinais de alterações no comportamento visual da criança frente a diferentes estímulos, servindo de alerta para o diagnóstico e a intervenção adequada. ${ }^{10}$ Este Método aplicou-se em estudos longitudinais em lactentes de alto risco Heloisa ${ }^{7,8,26}$, que avaliou a aplicabilidade do mesmo, detectando nesta ocasião algumas alterações no comportamento visual de lactentes prematuros.

No Brasil, dentre os instrumentos mais utilizados para avaliação do desenvolvimento motor tem-se a Escala canadense Alberta Infant Motor Scale (AIMS) que avalia o desempenho motor tanto de lactentes de risco como de lactentes com desenvolvimento típico. ${ }^{17,18}$

Entendendo que, apesar dos avanços no conhecimento na área de saúde neonatal a prematuridade continua sendo importante fator de risco para seqüelas sensório motoras ${ }^{8}$, sendo assim, o objetivo deste estudo é caracterizar o comportamento visual e o desenvolvimento motor de recém-nascidos pré-termo.

\section{MÉTODO}

Estudo descritivo, seccional no primeiro mês de idade corrigida, em uma coorte de RNPT, aninhado a um estudo amplo de acompanhamento no $1^{\circ}, 2^{\circ}$ e $3^{\circ}$ meses de vida de RNPT, do Projeto de Pesquisa, intitulado: "Comparação do comportamento visual no primeiro trimestre de vida de lactentes nascidos pré-termo em duas maternidades da cidade do Recife/PE". A população do estudo foi selecionada na maternidade do Hospital de Clínicas da Universidade Federal de Pernambuco HC/UFPE no período de julho de 2007 a abril de 2008, obedecendo aos seguintes critérios de inclusão: residentes na Região Metropolitana da cidade do Recife; nascido na maternidade do Hospital Escola, pré-termo com idade gestacional compreendida entre 28 semanas completas e 36 semanas e 6 dias ${ }^{1,2}$; de alta hospitalar ou internado, em condições clínicas favoráveis para realização das provas dos instrumentos de avaliação e assinatura do Termo de Consentimento Livre e Esclarecido pelos responsáveis para participação da criança no estudo.

Foram excluídos os lactentes que apresentaram lesão neurológica (hidrocefalia, hipertensão intracraniana/HIC grau III e IV, asfixia grave), apgar menor que 4 no $5^{\circ}$ minuto; malformação e/ou síndrome genética diagnosticada no período do estudo e infecção congênita confirmada e lactentes filhos de mães que apresentam alguma alteração mental que dificultasse a compreensão das perguntas realizadas no momento da seleção.

Foram desligados do estudo os lactentes internados em Unidade de Terapia Intensiva Neonatal durante o período das avaliações, os que apresentaram intercorrências neurológicas de qualquer natureza ou cujos responsáveis desistiram voluntariamente em participar do estudo.

Foram recrutados 989 recém-nascidos e 116 compuseram a população do estudo de aninhamento. Destes, 12 recém-nascidos obedeceram aos critérios do presente estudo, constituindo a amostra do mesmo.

Os dados referentes às variáveis biológicas da criança (Idade Gestacional, Peso ao Nascer, Sexo e Apgar) foram colhidos diretamente no prontuário dos lactentes. Já as variáveis maternas (Idade, Capacidade de Leitura, Escolaridade), as socioeconômicas e demográficas (renda familiar Per Capita, número de cômodos da residência, tipo de piso da resi- 
dência, coabitação dos pais, trabalho paterno e bens de consumo) foram respondidas pelo responsável da criança, através de questionário contendo perguntas fechadas, coletados pela equipe de recrutamento.

Durante a realização da coleta de dados, as mães foram esclarecidas sobre os objetivos e metodologia do estudo e, por meio da assinatura no termo de consentimento livre e esclarecido, autorizaram a participação de seus bebês na pesquisa, bem como a realização das fotos e da filmagem.

As avaliações foram realizadas no setor de Puericultura do Hospital Escola por terapeutas ocupacionais, devidamente treinadas. Cada lactente foi avaliado na presença de seu responsável, em sala apropriada, considerando o primeiro mês de vida no intervalo entre 7 dias antes da data de nascimento e 7 dias após a data de nascimento ${ }^{10}$.

O comportamento visuomotor dos RNPT foi avaliado por meio do Método de Avaliação da Conduta Visual de Lactentes, ${ }^{10}$ composto por 10 provas que qualificam as funções oculomotoras e apendiculares no primeiro trimestre de vida. Utiliza-se como material para avaliação, um aro vermelho suspenso por um cordão e a face do examinador. Cada prova pode ser aplicada em até 03 tentativas, com duração máxima de toda avaliação de 10 minutos.

As provas que compõem o método são seqüencialmente: fixação visual; contato de olho com o examinador; sorriso como resposta ao contato social; seguimento visual horizontal; seguimento visual vertical; exploração visual do ambiente; exploração visual da mão; aumento da movimentação de membros superiores ao visualizar o objeto; estender o braço na direção do objeto visualizado; sinais e sintomas oculares.

O registro das provas é baseado na observação direta dos componentes visuomotores, utilizando $\mathbf{S}$ (Sim) quando a criança apresenta o comportamento da resposta esperada,
$\mathbf{N}$ (não), quando não apresenta a resposta ao comportamento esperado e $\mathbf{O}$ (omitida) quando a aplicação das provas não é possível, devido a manifestações comportamentais negativas como, sono, choro, fome ou outro desconforto que leve a interrupção da avaliação ${ }^{10,11}$.

As adaptações, quanto à postura do RNPT durante a avaliação, foram realizadas com autorização da autora do Método de Avaliação da Conduta Visual de Lactentes. O lactente que apresentava peso inferior a $2.200 \mathrm{~g}$ era posicionado em decúbito dorsal sobre um suave encosto e quando seu peso era superior a $2.200 \mathrm{~g}$ era posicionado em decúbito dorsal sobre travesseiro anatômico tipo "caixa de ovo". Estas adaptações visaram favorecer a organização do bebê pela melhora da estabilidade postural e reduzir a chance de regurgitação do lactente, já que a inibição da atividade reflexa no RNPT depende da maturação do sistema nervoso central e a regurgitação é comum nos lactentes até o primeiro ano de vida, sendo maior quanto mais baixo peso for o $\mathrm{RN}^{19,20}$.

Para avaliação do desenvolvimento motor foi utilizada a escala padronizada Alberta Infant Movement Scale (AIMS) que incorpora o conceito neuromaturacional e as teorias dos sistemas dinâmicos. Trata-se de uma escala fidedigna, de fácil aplicação e capaz de diferenciar o desempenho motor normal do anormal de crianças nascidas a termo e pré-termo com idade corrigida, desde o nascimento até a idade da marcha independente $(18 \text { meses })^{18,21}$.

A criança deve ser observada em ambiente livre de obstáculos, com o mínimo de estimulação ou manipulação. A avaliação tem duração de 20 a 30 minutos e cada um dos comportamentos descritos nos diversos itens é registrado. A escala é constituída por 58 itens ou seqüências de movimentos, acompanhado por imagens que descrevem a postura do lactente, transferência de peso e movimentos antigravitacionais espontâneos em quatro posições posturais: decúbito dorsal e ventral, sentado e de pé ê, $18,22,23$. 
A cada item da prova observado é atribuído 1 ponto e cada item não observado é atribuído 0 ponto. $\mathrm{O}$ escore total é registrado pela soma dos pontos observados mais os pontos anteriores ao primeiro item observado no período de desenvolvimento em que o lactente se encontra em cada subescala. O escore total e a idade corrigida determinam a posição do lactente em cada uma das curvas percentilares (entre percentil 5 a 90) desenvolvidas com a amostra normativa canadense $\mathrm{e}^{17,21,23-24}$.

Os dados coletados foram digitados no software Epi-Info 2004, todas as respostas foram pré-codificadas e checadas, e posteriormente foi procedida à entrada dos dados no programa. A análise foi realizada por meio de frequência simples. O estudo foi aprovado pelo Comitê de Ética em Pesquisa do Hospital das Clínicas da Universidade Federal de Pernambuco (HC/UFPE), sob o ${ }^{\circ} 136$, em dezembro de 2006.

\section{RESULTADOS}

A amostra foi constituída por 12 recémnascidos pré-termo (RNPT), avaliados no primeiro mês de idade corrigida. Na tabela 1 ob-

Tabela 1: Variáveis biológicas dos recém-nascidos prematuros nascidos na Maternidade do Hospital das Clínicas da Universidade Federal de Pernambuco - Recife, PE

\begin{tabular}{lcc}
\hline \multicolumn{1}{c}{ VARIÁVEL } & \multicolumn{2}{c}{ POPULAÇÃO } \\
& n & $\mathbf{1 2}$ \\
\hline Sexo & 10 & \% \\
Masculino* & 2 & 83,3 \\
Feminino & & 16,6 \\
Idade gestacional & 1 & \\
28 semanas a 32 semanas e 6 dias & 11 & 8,3 \\
33 semanas a 36 semanas e 6 dias & & \\
Peso ao nascer & 9 & 75 \\
1.500 a 2.499 & 3 & 25 \\
$>2.500$ & 12 & 100 \\
Apgar & & \\
$>7$ ( no 5 ${ }^{\circ}$ minuto) & & \\
$*$ 1 caso de gemelaridade & &
\end{tabular}

serva-se as variáveis biológicas e na tabela 2 as variáveis socioeconômicas e demográficas da amostra.

Tabelas 2: Variáveis socioeconômicas e demográficas dos recém-nascidos prematuros nascidos na Maternidade do Hospital das Clínicas da Universidade Federal de Pernambuco - Recife, PE (2007/2008)

\begin{tabular}{|c|c|c|}
\hline \multirow{2}{*}{ VARIÁVEL } & \multicolumn{2}{|c|}{$\begin{array}{c}\text { POPULAÇÃO } \\
n=12\end{array}$} \\
\hline & $\mathbf{n}$ & $\%$ \\
\hline \multicolumn{3}{|l|}{ Idade materna (anos) } \\
\hline 18 a 20 & 2 & 16,7 \\
\hline 21 a 31 & 10 & 83 \\
\hline \multicolumn{3}{|c|}{ Escolaridade materna (anos) } \\
\hline $1^{\circ}$ grau maior incompleto & 5 & 41,6 \\
\hline $1^{\circ}$ grau maior completo & 2 & 16,6 \\
\hline $2^{\circ}$ grau incompleto & 1 & 8,3 \\
\hline $2^{\circ}$ grau completo & 4 & 33,3 \\
\hline \multicolumn{3}{|c|}{ Mãe convive com o pai da criança } \\
\hline Sim & 11 & 91,6 \\
\hline Não & 1 & 8,3 \\
\hline \multicolumn{3}{|l|}{ Trabalho materno } \\
\hline $\operatorname{Sim}$ & 5 & 41,6 \\
\hline Não & 7 & 58,4 \\
\hline \multicolumn{3}{|l|}{ Trabalho paterno } \\
\hline Sim & 6 & 50 \\
\hline Não & 6 & 50 \\
\hline \multicolumn{3}{|c|}{ Renda mensal**(Salário mínimo) } \\
\hline$<1$ salário & 6 & 50 \\
\hline$>1$ a 2 salários & 5 & 41,6 \\
\hline$>2$ salários & 1 & 8,3 \\
\hline \multicolumn{3}{|c|}{ Coabitação (menos de 4 pessoas) } \\
\hline $\operatorname{Sim}$ & 5 & 41,6 \\
\hline Não & 7 & 58,4 \\
\hline \multicolumn{3}{|l|}{ Residência (cômodos) } \\
\hline Até 3 & 4 & 33,3 \\
\hline Mais de 3 & 8 & 66,6 \\
\hline \multicolumn{3}{|l|}{ Canalização sanitária } \\
\hline Sim & 12 & 100 \\
\hline \multicolumn{3}{|l|}{ Água encanada } \\
\hline $\operatorname{Sim}$ & 12 & 100 \\
\hline \multicolumn{3}{|l|}{ Energia elétrica } \\
\hline Sim & 12 & 100 \\
\hline \multirow{2}{*}{\multicolumn{3}{|c|}{$\begin{array}{l}\text { Bens de Consumo } \\
\text { (televisão, DVD, } \\
\text { geladeira e celular) }\end{array}$}} \\
\hline & & \\
\hline Sim & 12 & 100 \\
\hline
\end{tabular}

Quanto ao comportamento visual dos RNPT, todos responderam positivamente as provas 1 e 2 (fixação visual e contato visual com examinador). Nas demais provas foram observadas as respostas com: $8,3 \%$ ao sorriso como resposta ao contato social 


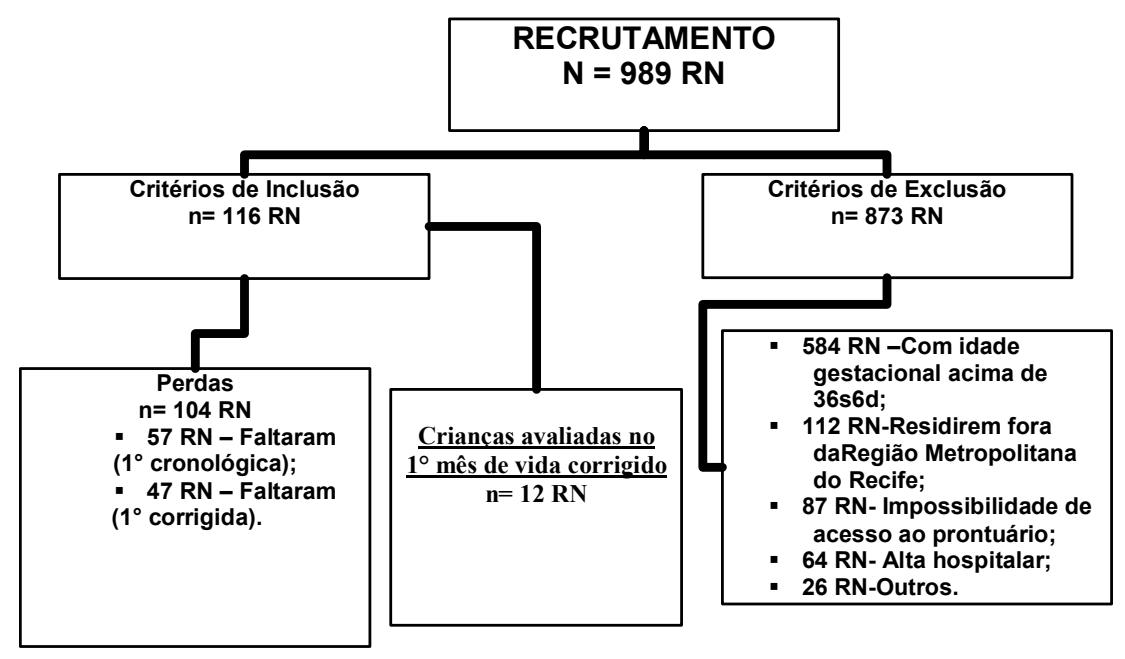

Figura 1: Recrutamento dos recém-nascidos prematuros na Maternidade do Hospital das Clínicas da Universidade Federal de Pernambuco - Recife, PE (2007/2008).

(prova 3 ); $83,3 \%$ ao seguimento visual horizontal (prova 4); $50 \%$ ao seguimento visual vertical (prova 5); 91,6\% a exploração visual do ambiente (prova 6); $8,3 \%$ ao aumento da movimentação de membros superiores (prova 8).

Nas provas 7 e 9 (exploração visual da mão e estender o braço na direção do objeto visualizado) apresentaram freqüência nula. Dos $41 \%$ dos sinais oculares evidenciados, $33 \%$ são desvios oculares e $8 \%$ são presença de secreção ocular.

Já em relação ao desenvolvimento motor, apresentaram percentil abaixo de $5(25 \%)$, percentil $5(8,3 \%)$, percentil $10(8,3 \%)$, percentil $25(25 \%)$, percentil $50(8,3 \%)$ e percentil $75(25 \%)$.

\section{DISCUSSÃO}

Os RNPT apresentaram fator de risco biológico (prematuridade), porém os fatores socioeconômico-demográficos mostraram-se adequados podendo funcionar como fator de proteção no desenvolvimento infantil. Halpern et al ${ }^{25}$ e Mancini ${ }^{16}$ corroboram com esses acha- dos, já que descrevem a interação dos fatores biológicos e ambientais como influenciadores do desenvolvimento infantil podendo ser favoráveis ou desfavoráveis ao mesmo.

No que se refere ao comportamento visual, os RNPT exibiram respostas semelhantes a outros estudos com prematuros e a termo, que trazem as provas 1, 2, 4 e 6 como esperadas para esta faixa etária ${ }^{7}$, com exceção da prova 3 (sorriso em resposta ao contato social) ${ }^{10,26}$.

Apesar do contato de olho estar presente nos lactentes estudados e ser determinante para estabelecer a reciprocidade e comunicação entre mãe e bebê, que é manifestada pelo do sorriso ${ }^{27}$, na prova 3 , que avaliou a expressão do sorriso em resposta ao estímulo, verificou-se freqüência inferior ao referido na literatura tanto para lactentes a termo ${ }^{10}$ como para os pré-termo ${ }^{8,28}$.

Destaca-se que o sorriso como resposta ao rosto humano, demonstra a capacidade do bebê de expressar-se afetivamente, constituindo uma realização social vital ${ }^{29}$. Além disso, seu desenvolvimento interativo e intensificação em bebês parecem ser dependentes de experiências mediadas pela interação visual ${ }^{30}$. 
No entanto, os dados referentes a esses achados não são conclusivos e recomendamse estudos prospectivos para melhor compreensão sobre o tema.

Na prova 5 apresentaram baixa freqüência de respostas, como mostra os estudos de Carvalho ${ }^{26}$ e Gagliardo, Gonçalves e Lima. ${ }^{8,10}$ Segundo Hyvärinen ${ }^{31}$ o seguimento visual vertical se desenvolve posteriormente, apresentando maior freqüência no $2^{\circ}$ mês. Nas provas $7 \mathrm{e}$ 9 , com freqüência nula e a prova 8 com freqüência reduzida, corroboram com a literatura, já que estes comportamentos são previstos para o $3^{\circ} / 4^{\circ}$ mês s, $^{7,26,261}$.

Com relação ao desenvolvimento motor, $41,6 \%$ apresentaram abaixo do percentil 10 , indicando assim um sinal de alerta, mesmo com a idade corrigida, pois ainda estão aquém dos bebês a termo no que se refere ao desenvolvimento motor. Campos et al ${ }^{17}$ e Piper e Darrah ${ }^{18}$ comentam que o percentil do teste indica quanto mais alto menor a chance de apresentar atrasos no desenvolvimento. Contudo, a interpretação de baixos percentis, não é clara, indicando assim um alerta ao desenvolvimento no percentil 10 ou abaixo.

A correção da idade para 40 semanas (termo) ao utilizar o Método de Avaliação da Conduta Visual e do AIMS em RNPT foi um fator importante neste estudo que facilitou a compreensão das aquisições observadas dentro da faixa etária esperada.

Os recém-nascidos prematuros estão mais predispostos a desenvolver alterações oftalmológicas, podendo apresentar alterações no seu desenvolvimento global, já que a visão interfere no desenvolvimento dos outros sistemas sensoriais, no desenvolvimento motor e nos aspectos cognitivo e sócio-emocional. ${ }^{7,10}$ Contudo, as alterações oculares observadas nos lactentes prematuros do presente estudo podem ser transitórias, decorrentes da própria imaturidade. Dessa maneira, esses lactentes perma- neceram em seguimento para investigação visto que, apesar das alterações, os RNPT do estudo apresentaram comportamento visual esperado para faixa etária. Porém no que se refere às aquisições do desenvolvimento motor, mesmo com a idade corrigida ainda estão aquém dos bebês a termo.

$\mathrm{O}$ atraso motor pode ter influência dos fatores de risco (idade gestacional), já que estudos afirmam que o desenvolvimento motor dos prematuros normalmente apresenta alterações do tono muscular, reflexos primitivos ausentes ou diminuídos e movimentos espontâneos reduzidos, conforme o grau de prematuridade ${ }^{3,7,9}$, pois apresentaram condições socioeconômicas e demográficas adequadas.

Por fim, foi possível observar que o atraso motor pode ter influência dos fatores de risco biológicos. Assim, a utilização do Método de Avaliação da Conduta Visual e da AIMS em RNPT alcançou o objetivo de observar precocemente atrasos nas funções visuais/motoras que influenciam no desenvolvimento normal, de acordo com correção da idade, além de apresentar considerações sobre a necessidade de teste de triagem, para que intervenções sejam realizadas em tempo oportuno. Já que à relação do desenvolvimento visual e motor estão associados e quando um apresenta um atraso interferirá na ação do outro, dificultando no repertório funcional e qualidade de vida dessas crianças.

\section{AGRADECIMENTOS}

Ao CNPq que financiou através do edital Universal 2006/2008 este projeto, ao Hospital das Clínicas/UFPE, aos assistentes de pesquisa que participaram da equipe de recrutamento e avaliação, e aos familiares dos recém-nascidos prematuros que participaram desta pesquisa. 


\section{REFERÊNCIAS}

1. Spacilli, MDB, Chiea, MA, Albuquerque, PB, Bittar, RE, Zugaib, M. Estudo de algumas variáveis maternas relacionadas com a prematuridade. Rev. Med. HU-USP. 2000; 10(1): 19-23.

2. Filho, NA, Correâ, MD. Manual de Perinatologia. MEDSI: Rio de Janeiro, 1995.

3. Ayche MG, Coríntio MN. Considerações sobre o desenvolvimento motor do prematuro. Temas sobre desenvolvimento. 2000;12, (71): 5-9.

4. Rugolo, LMSS. Crescimento e desenvolvimento a longo prazo do prematuro extremo. J Pediatr. 2005; 81(1 Supl):101-110.

5. Graziano, RM, Leone, CR. Frequent ophthalmologic problems and visual development of preterm newborn infants. J de Pediatr 2005;81(1):95-100.

6. Ruas, TCB, Ravanini, SG, Martinez, CS; Gagliardo, HR; Françoso, MFC; Rim, PHH. Avaliação do comportamento visual de lactentes no primeiro e segundo meses de vida. Rev. Bras. Crescimento Desenvolv. Hum. 2006; 16(3): 1-8.

7. Ruas, TCB, et al. A comparação de funções apendiculares desencadeadas pela visão em lactentes nascidos pré-termo e a termo no primeiro trimestre de vida. Rev. Bras. Cresc. e Desenv. Hum. 2010; 20(3): 680-87.

8. Albuquerque, $\mathrm{RC}$, et al. Comportamiento visuomotor de lactantes pretermino en el primer mes de vida comparacion entre las edades cronologica y corregida. Rev. Neurol. 2009; 48 (1): 13-16.

9. Mota, LA, Sá, FE, Frota, MA. Estudo comparativo do desenvolvimento sensório-motor de recém-nascido prematuros da unidade de terapia intensiva neonatal e do método canguru. RBPS 2005; 18(4): 191-198.

10. Gagliardo, HGRG, Gonçalves, VMG, Lima, MCMP. Método para avaliação da conduta visual de lactentes. Arq de NeuroPsiquiatria 2004; 62(2-A): 300-306.

11. Gagliardo, HGRG, Gabbard, C, Gonçalves, VMG. Coordenação visuomotora em lactentes de baixo peso ao nascimento: revisão da literatura. Temas sobre Desenvolvimento $2002 ; 11(62): 51-55$.

12. Mancini MC et al. Estudo do desenvolvimento da função motora aos 8 e 12 meses de idade em crianças pré-termo e a termo.Arq de Neuro-Psiquiatria 2002;60(4):974-980.

13. Ribeiro, MVLM. Avaliação clínica do recém-nascido a termo. In: Ribeiro, MVLM, Gonçalves, VMG. Neurologia do Desenvolvimento da Criança. REVINTER: Rio de Janeiro; 2006.

14. Lima MC et al. Determinants of mental and motor development at 12 months in a low income population: a cohort study in northeast Brazil. Acta Pediatr 2004;93(7):969-975.

15. De Andraca I, Pino P, De la Parra A, Riveira F, Castillo M. Risk factors for psychomotor development among infants born under optimal biological conditions. Rev Saúde Pública 1998; 32: 138-47.

16. Mancini $M C$, Megale L, Brandão MB, Melo APM, Sampaio RF. Efeito moderador do risco social na relação entre risco biológico e desempenho funcional infantil. Rev Bras Saúde Materno Infantil 2004;4(1):25-34.

17. Campos D et al. Agreement between scales for screening and diagnosis of motor development at 6 months. J. Pediatr 2006; 82(6):470-474.

18. Piper, M, Darrah, J. Motor assesment of the developing infant. Philadelphia: Saunders; 1994.

19. Costa AJF, Silva GAP, Gouveia PAC, Filho EMP. Prevalência de refluxo gastroesofágico patológico em lactentes regurgitadores. J Pediatr 2004; 80(4): 291295. 
20. Olhweiler L, Silva AR, Rotta NT. Estudo dos reflexos primitivos em pacientes recém-nascidos pré-termo normais no primeiro ano de vida. Arq Neuro-Psiquiatra 2005; 63(2-A): 294-297.

21. Jeng SF, Yau KIT, Chen LC, Hsiao SF. Alberta Infant Motor Scale: Reliability and Validity When Used on Preterm Infants in Taiwan. Phys Ther 2000;80(2):168-178.

22. Manacero, S, Nunes, ML. Evaluation of motor performace of preterm newborns during the first months of life using the Albert Infant Motor Scale (AISM). J Pediatr.2008;84(1):53-58.

23. Piper MC,Pinnell LE, Darrah J, Maguire T,Byrne PJ. Construction and validation of the Albert Infant Motor Scale (AIMS). Can J Public Health 1991;83(2):46-50.

24. Almeida KM, Dutra MVP, Mello RS, Reis ABR, Martins OS. Validade concorrente e confiabilidade da Albert Infant Motor Scale em lactentes nascidos prematuros. J Pediatr 2008; 84(5): 442-448.

25. Halpern, R., Giugliani ERJ, Victora CG, Barros FC, Horta BL. Fatores de risco para suspeita de atraso no desenvolvimento neuropsicomotor aos 12 meses de vida. J Pediatr 2000; 76(6): 421-428.
26. Carvalho, BGE. Triagem visual de bebês prematuros: verificação da aplicabilidade do Método de Avaliação da Conduta Visual de Lactentes [tese]. São Carlos: Universidade Federal de São Carlos; 2005.

27. Ribas, AFP, Seidl de Moura ML. Interação precoce mãe-bebê. Cadernos de Psicologia. 1998. 9:50-66.

28. Albuquerque, RC, Cabral-Filho, JE, Gagliardo, HGRG. Comparação do Comportamento Visual entre as Idades Cronológica e Corrigida de Lactentes Pré-termo no $1^{\circ}$ Mês de Vida. In: Congresso Brasileiro de Ensino e Pesquisa em Saúde da Criança e do Adolescente, 2008. Revista de Pediatria SOPERJ, 2008. v. 1.

29. Souza EAP, Mello BBA. Desenvolvimento infantil: uma visão comportamental. In: Moura-Ribeiro MVL, Gonçalves VMG (Org). Neurologia do desenvolvimento da criança. Revinter.2006: 313-28.

30. Messinger D, Fogel A. The interactive development of social smiling. Adv Child Dev Behav. 2007;35:327-66.

31. Hyvarinen, L.Considerations in evolutioin e treatment of the child with low vision. American Journal Occupational Therapy 1995; 49(9):891-7.

Recebido em: 08/ago./2010

Modificado em 26/nov./2010 Aceito em 16/mar./2011 